\title{
Chance der Entwicklung
}

Die in der österreichischen Psychiatrie und Psychotherapie tätigen Menschen verschiedenster Berufsgruppen haben mit dem Tod von Univ.-Prof. Dr. Hans Georg Zapotoczky einen großen Lehrer und wichtigen Impulsgeber verloren. Ich hatte das Glück, auf seiner verhaltenstherapeutischen Station im AKH die ersten zwei Jahre meiner Facharztausbildung mitzuarbeiten. So lernte ich Psychiatrie und Psychotherapie nicht nur durch Fragebögen, standardisierte Interviews, Diagnosemanuals oder Konsensuskonferenzen kennen, sondern durch viele hundert, gemeinsam in der großen Visitenrunde auf Station 05B besprochene, Einzelschicksale.

Die Betonung des Individuellen des betroffenen Patienten vor dem Gemeinsamen irgendeiner Diagnosekategorie war Methode. In möglichst ganzheitlicher Betrachtung wurde versucht, den Leidensweg jedes einzelnen Patienten nachzuvollziehen und wenigstens ansatzweise zu verstehen. Diese Grundeinstellung war getragen vom Optimismus, in jedem einzelnen Fall eine Weiterentwicklung zu ermöglichen und die persönlichen Stärken zur Gesundung zu nutzen. Die Chance der Entwicklung von Menschen jeden Lebensalters und jeder Diagnose war das zentrale Credo von Hans Georg. Deshalb stellte er viele Fragen - sowohl an die Patienten als auch an uns -, die in Lehrbüchern nicht zu finden sind. Dabei berührte er viele Bereiche des Lebens, die nicht nur psychiatrisch-psychotherapeutische, sondern auch kulturelle, soziologische, philosophische oder religiöse Inhalte - eben den Patienten als Ganzes - betrafen.

"Im Leben des Menschen ist alles Entwicklung."

Hans Georg Zapotoczky

Es ist unmöglich, Hans Georg Zapotoczky als Herausgeber der von inm gemeinsam mit Univ.-Prof. Dr. Pöldinger gegründeten Psychopraxis zu ersetzen. Vielmehr soll sich die Zeitschrift im Sinne von Hans Georg weiterentwickeln. Besonders die wunderbaren, und in dieser Dichte nur in der Psychiatrie und Psychotherapie möglichen, Erfahrungen sollen in individuellen Schicksalen nachgezeichnet werden. Dabei soll nicht nur das kleinste gemeinsame Vielfache vieler Patienten mit einer spezifischen Diagnose herausgestrichen werden, sondern es soll auch aufgezeigt werden wie im komplexen Einzelfall nachgedacht, diagnostiziert, behandelt und Entwicklungen gefördert werden können. Ich hoffe, dass dies mir, dem Herausgeberteam, dem wissenschaftlichen Beirat und unseren Autoren gut gelingen wird.

\section{Peter Fischer}

